12 EFFICACY OF EARLY CONTROLLED MOTION OF THE ANKLE IN NON-OPERATIVE TREATMENT OF ACUTE ACHILLES TENDON RUPTURE. AN ASSESSOR-BLINDED RCT

${ }^{1}$ Kristoffer Weisskirchner Barfod*, ${ }^{2}$ Maria Swennergren Hansen, ${ }^{1}$ Per Hölmich, ${ }^{2}$ Morten Tange Kristensen, ${ }^{3}$ Anders Troelsen. ${ }^{1}$ Sports Orthopedic Research Center - Copenhagen (SORC-C), Arthroscopic Center, Department of Orthopedic Surgery, Copenhagen University Hospital, Amager-Hvidovre, Denmark; ${ }^{2}$ Physical Medicine and Rehabilitation Research Copenhagen (PMR-C),Department of Physiotherapy and Occupational Therapy, Copenhagen University Hospital Hvidovre, Denmark; ${ }^{3}$ Department of Orthopaedic surgery, Clinical Orthopaedic Research Hvidovre, Copenhagen University Hospital Hvidovre, Denmark

10.1136/bjsports-2019-scandinavianabs.12

Background Early controlled ankle motion (ECM) is widely used in the non-operative treatment of acute Achilles tendon rupture although its safety and efficacy has not been investigated properly in a randomized setup.

Purpose/aim of the study To investigate if ECM of the ankle was superior to immobilization (IM) in the treatment of acute Achilles tendon rupture.

Materials and methods The study was performed as an assessor blinded randomized controlled trial with patients allocated in a 1:1 ratio to one of two parallel groups. Patients aged 18 to 70 years were eligible for inclusion. The ECM group performed movements of the ankle 5 times a day from week 3 to 8 after rupture. The control group was immobilized (IM). The primary outcome was the Achilles tendon Total Rupture Score (ATRS) evaluated at 1 year post-injury. Secondary outcomes were: heel-rise-work test (HRW), Achilles tendon elongation and rate of re-rupture. Analysis was conducted as intention-to-treat with imputation of missing data.

Findings/results 189 patients were assessed for eligibility and 130 included from February 2014 to December 2016; 64 ECM and 58 IM. There was no statistically significant differences $(p>0.3)$ between the ECM and the IM groups at 1 year; Mean (SD) ATRS was 74 (18) and 75 (18), respectively. HRW was $60 \%$ (21) and $60 \%$ (21) of the uninjured limb, and elongation was $18 \mathrm{~mm} \mathrm{(13)} \mathrm{and} 16 \mathrm{~mm}$ (11), respectively. Correspondingly, there were 6 and 7 re-ruptures. Conclusions ECM revealed no benefit to IM in any of the investigated outcomes.

\section{DEEP VEIN THROMBOSIS AFTER ACUTE ACHILLES TENDON RUPTURE. AN RCT COMPARING EARLY CONTROLLED MOTION OF THE ANKLE WITH NO MOTION}

${ }^{1}$ Kristoffer Weisskirchner Barfod*, ${ }^{2}$ Emil Nielsen, ${ }^{2}$ Beth Hærsted Olsen, ${ }^{2}$ Pablo Gustavo Vinicoff, ${ }^{3}$ Anders Troelsen, ${ }^{1}$ Per Holmich. 'Sports Orthopedic Research Center Copenhagen (SORC-C), Arthroscopic Center, Department of Orthopedic Surgery, Copenhagen University Hospital, Amager-Hvidovre, Denmark; ${ }^{2}$ Department of Radiology, Copenhagen University Hospital, Amager-Hvidovre, Denmark; ${ }^{3}$ Department of Orthopaedic surgery, Clinical Orthopaedic Research Hvidovre, Copenhagen University Hospital Hvidovre, Denmark

\subsection{6/bjsports-2019-scandinavianabs.13}

Background Deep vein thrombosis (DVT) following acute Achilles tendon rupture (ATR) is common (up to 34\%) and potentially dangerous. Immobilization (IM) is thought to be an important factor in the pathogenesis.

Purpose of the study To investigate if early controlled ankle motion ECM could reduce the incidence of DVT compared to IM in the treatment of acute Achilles tendon rupture.
Materials and methods The study was performed as a randomized controlled trial. Patients aged 18 to 70 years were eligible for inclusion. Treatment was non-operative. The ECM group performed movements of the ankle 5 times a day from week 3 to 8 after rupture. The control group was IM for 8 weeks. Follow up was performed with Color Doppler ultrasound at 2 and 8 weeks by two experienced radiologists. DVT was a secondary outcome, why a secondary power calculation was performed: 124 patients were required to have a $60 \%$ chance of detecting, as significant at the $5 \%$ level, a decrease in DVT from $34 \%$ in the IM group to $17 \%$ in the ECM group.

Findings/results 189 patients were assessed for eligibility and 130 randomized: 68 (ECM-group) and 62 (IM-group). All patients participated in the follow up. 62 (47.7\%) patients were diagnosed with DVT; 34/69 (49.3\%) in the ECM group and $28 / 61(45.9 \%)$ in the IM group $(p=0.70)$.

Conclusions The incidence of asymptomatic DVT was higher than previously reported as $48 \%$ presented with DVT. ECM revealed no benefit to IM in reducing the incidence of DVT.

\section{INDIVIDUAL TREATMENT SELECTION FOR ACUTE ACHILLES TENDON RUPTURE BASED ON THE COPENHAGEN ACHILLES LENGTH MEASUREMENTS (CALM)}

${ }^{1}$ Kristoffer Weisskirchner Barfod*, ${ }^{2}$ Maria Swennergren Hansen, ${ }^{1}$ Håkon Sandholdt, ${ }^{1}$ Anders Boesen, ${ }^{1}$ Per Holmich, ${ }^{3}$ Anders Troelsen, ${ }^{2}$ Morten Tange Kristensen. ${ }^{1}$ Sports Orthopedic Research Center - Copenhagen (SORC-C), Arthroscopic Center, Department of Orthopedic Surgery, Copenhagen University Hospital, Amager-Hvidovre, Denmark; ${ }^{2}$ Physical Medicine and Rehabilitation Research - Copenhagen (PMR-C),Department of Physiotherapy and Occupational Therapy, Copenhagen University Hospital, Amager-Hvidovre, Denamrk; ${ }^{3}$ Clinical Orthopedic Research Hvidovre (CORH), Department of Orthopedic Surgery, Copenhagen University Hospital, Amager-Hvidovre, Denmark

\subsection{6/bjsports-2019-scandinavianabs. 14}

Background Acute Achilles tendon rupture (ATR) can be treated operatively or non-operatively. An evidence based selection tool is needed to guide choice of treatment.

Purpose To investigate if treatment selection in patients with ATR can be guided by Amlang's ultrasound classification (AmC) or the Copenhagen Achilles Length Measurement (CALM).

Methods The study was performed as a prospective cohort study. Patient were 18 to 70 years and treated non-operatively. AmC and CALM were performed at baseline and correlated to outcome at 1 year. The primary outcome was the Achilles tendon Total Rupture Score (ATRS). Secondary outcomes were: heel-rise-work test, re-rupture rate and CALM at 1 year. ROC analysis was performed to determine a cut off for acceptable elongation of CALM at baseline given that elongation at 1 year was not to exceed $10 \%$. ClinicalTrial.gov Identifier: NCT02062567.

Results CALM was performed at baseline in 130 patients and AmC in 109. AmC showed no statistically significant correlation to any outcome parameter. CALM at baseline correlated to CALM at 1 year $\mathrm{r}=0.214(\mathrm{p}<0.01)$. The ROC model had AUC $=0.67$. An elongation of $7 \%$ at baseline had a sensitivity of 0.77 and specificity of 0.50 .

Conclusions Elongation of the Achilles tendon at baseline measured with CALM was weakly correlated to elongation at 1 year follow up suggesting that CALM at base line can 